\title{
Supernova OH (1720 MHz) masers in Sgr A East, W28 and G359.1-0.5
}

\author{
F. Yusef-Zadeh
}

Department of Physics and Astronomy, Northwestern University, Evanston, IL. 60208

D.A. Roberts

Department of Physics and Astronomy, Northwestern University, Evanston, IL. 60208

Geoff Bower

UC Berkeley, Berkeley, CA 94720

M. Wardle

School of Physics, University of Sydney, NSW 2006, Australia

W. M. Goss

NRAO, P.O. Box 0, Socorro, New Mexico 87801

\begin{abstract}
.
A new class of $\mathrm{OH}(1720 \mathrm{MHz})$ masers unaccompanied by main-line transitions have recently been discovered (Frail, Goss and Slysh 1994). These masers lie at the interface between supernova remnants (SNRs) interacting with molecular clouds. We discuss three new aspects of SN masers found in the direction toward the Galactic center: (i) the detection of a new $-130 \mathrm{~km} \mathrm{~s}^{-1} \mathrm{OH}(1720 \mathrm{MHz})$ maser in the southern lobe of the molecular ring at the Galactic center, (ii) the detection of extended $\mathrm{OH}$ $(1720 \mathrm{MHz})$ maser emission from W28 accompanying the compact maser sources and (iii) the detection of linear polarization of the brightest $\mathrm{OH}$ $(1720 \mathrm{MHz})$ maser in SNR G359.1-0.5.
\end{abstract}

\section{Introduction}

It is increasingly evident that the new class of so-called "supernova masers" is distinguishing itself as an excellent signature of the interaction of SNR's and molecular clouds. In addition, there is now considerable theoretical and observational evidence that $\mathrm{OH}(1720 \mathrm{MHz})$ maser spots probe the physical condition of postshock gas which results from the expansion of a SNR driving a shock into a molecular cloud as discussed in the conference. The study of a number of SN masers have led to some of the first measurements of postshock magnetic field. 
There are a total of 20 known supernova masers in the Galaxy, the largest concentration of which is observed toward the center of the Galaxy (Yusef-Zadeh et al. 1999). The reason for such a concentration is not clear but high molecular density ranging between $10^{4}$ and $10^{5} \mathrm{~cm}^{-3}$ combined with $\mathrm{OH}$ column density of $10^{16}-10^{17} \mathrm{~cm}^{-2}$ (Lockett et al. 1999) as well as a high concentration of SNRs in this region are needed to produce the larger number of SN masers observed in the Galactic center region. Here we discuss some new characteristics of three different SN masers located between us and the Galactic center.

\section{Sgr A East: the Blue-shifted OH Feature}

Perhaps the most complicated group of SN masers in the Galaxy is the one located at the Galactic center. A clumpy molecular ring (also known as the Circumnuclear Disk or CND), on a scale of 2 to 5 pcs is circling the Galactic center with a rotational velocity of about $100 \mathrm{kms}^{-1}$ (see Jackson et al. 1993). On a larger scale of up to 15 pcs, SNR G0.0+0.0 or Sgr A East is known to be dynamically coupled to the $50 \mathrm{~km} \mathrm{~s}^{-1}$ molecular cloud. The detection of several $\mathrm{OH}(1720 \mathrm{MHz})$ maser spots at velocities ranging between 43 and $66 \mathrm{kms}^{-1}$ surrounding the Sgr A East shell was first reported in 1996. These observations supported the picture of the physical interaction of Sgr A East and the $50 \mathrm{~km} \mathrm{~s}^{-1}$ molecular cloud (Yusef-Zadeh et al. 1999). These studies show another group of $\mathrm{OH}(1720 \mathrm{MHz})$ masers to the northeast lobe of the molecular ring at the velocity of $+134 \mathrm{~km} \mathrm{~s}^{-1}$. The inferred magnetic field strength along the line of sight due to Zeeman splitting is between 2 and $5 \mathrm{mG}$. The orientations of the magnetic fields along the line of sight are away from the Earth for all masers except for the $+134 \mathrm{~km} \mathrm{~s}^{-1}$ CND masers, which are directed toward the Earth. The cluster of highly red-shifted $\mathrm{OH}(1720 \mathrm{MHz})$ masers associated with the CND implies that the Sgr A East SNR shell is also expanding into the molecular ring and is responsible for the production of the $\mathrm{OH}(1720 \mathrm{MHz})$ maser emission behind shock front. Recent molecular $\mathrm{H}_{2}$ observations of the Sgr A East masers supports the picture in which $\mathrm{OH}(1720 \mathrm{MHz})$ masers are tracing post-shocked gas The presence of such a cluster of $\mathrm{OH}(1720 \mathrm{MHz})$ masers at $+134 \mathrm{kms}^{-1}$ provides compelling evidence that Sgr A East is indeed very close to the Galactic center and is interacting with the molecular ring circling the center of the Galaxy.

An even more striking result was realized when we analyzed the archival $\mathrm{OH}(1720 \mathrm{MHz})$ data taken in 1986 based on A-array VLA observation of the Galactic center with a spatial and spectral resolutions of $4.4^{\prime \prime} \times 3.6^{\prime \prime}$ and 8.6 $\mathrm{kms}^{-1}$, respectively. This 1986 observation confirmed all the bright $\mathrm{OH}(1720$ $\mathrm{MHz}$ ) maser spots which were observed about ten years later in 1996. Understandably the class of SN masers could have been discovered serendipitously several years earlier than the rediscovery paper by Frail, Goss and Slysh in (1994) which was published 26 years after the detection of masers toward SNRs (e.g., Goss 1968). Another interesting aspect of the 1986 data is the detection of a highly blue-shifted $\mathrm{OH}(1720 \mathrm{MHz})$ maser line at the velocity of $-131.6 \mathrm{~km} \mathrm{~s}^{-1}$ at $\alpha=17^{\mathrm{h}} 42^{\mathrm{m}} 28 .{ }^{\mathrm{s}} 03, \delta=-29^{\circ} 00^{\prime} 6 .{ }^{\prime \prime} 4$. This maser has not been detected since then in spite of efforts made recently by M. Goss. The spectrum of this new maser has a peak flux density of $75 \mathrm{mJy}$ with the rms noise of $6.6 \mathrm{mJy}$ beam $^{-1}$. The peak flux is 4 times brighter than the $3 \sigma$ upper limit of $16 \mathrm{mJy}$ from the 
1996 epoch observations. Figure 1a shows the positions of the symmetrically placed blue- and red-shifted $\mathrm{OH}$ masers as crosses and are superposed on the molecular ring traced by $\mathrm{H}_{2}$ emission. The velocity of the masers is consistent with the sense of rotation of the molecular ring. We believe the $\pm 132 \mathrm{kms}^{-1}$ velocity features are signifying the true rotational velocity of the molecular gas orbiting the Galactic center. This is because the largest amplification occurs in the direction of largest velocity coherence which tends to be perpendicular to the shock front as it accelerates the gas in the transverse direction. This implies that the velocity of masers reflects the systemic motion of the molecular cloud. If so, the mass enclosed within a radius of about $45^{\prime \prime}(1.8 \mathrm{pc}$ at the distance of 8.5 kpc) from $\mathrm{Sgr} \mathrm{A}^{*}$ is estimated to be about $7 \times 10^{6} M_{\odot}$ using Keplerian motion. We can also interpret that median velocity of the $\mathrm{OH}(1720 \mathrm{MHz})$ masers in the ring is consistent with no radial motion of the Local Standard of Rest with respect to the Galactic center.

\section{W28: Extended Maser Emission}

Studies of the inner several degrees of the Galactic plane toward the Galactic center detected extended $\mathrm{OH}(1720 \mathrm{MHz})$ emission from G359.1-0.5, G357.7+0.3 (The Square Nebula) and G357.7-0.1 (the Tornado Nebula) (Yusef-Zadeh et al., 1995; 1999). The largest extended $\mathrm{OH}(1720 \mathrm{MHz})$ emission arises from G357.7+0.3 distributed over an angular size of $>15^{\prime}$ corresponding to $27 \mathrm{pc}$ at the distance of $6.4 \mathrm{kpc}$ (Leahy 1989). More recently, we observed W28 at the distance of $2 \mathrm{kpc}$ and detected new extended $\mathrm{OH}(1720 \mathrm{MHz})$ features coincident with the compact maser spots at similar radial velocities. Figure 1b shows contours of extended emission superposed on the $\lambda 20 \mathrm{~cm}$ continuum image of W28 with a resolution of $28^{\prime \prime} \times 16^{\prime \prime}$. It is clear that the extended $\mathrm{OH}(1720$ $\mathrm{MHz}$ ) emission follows the continuum morphology.

These features are probably low-gain masers that co-exist with the bright, compact masers, as has been argued in the case of G359.1-0.5 (Yusef-Zadeh et al. 1995). Extended $\mathrm{OH}(1720 \mathrm{MHz}$ ) emission may in principle be produced by thermal foreground objects in a manner analogous to the widespread Galactic $\mathrm{OH}(1720 \mathrm{MHz})$ emission observed in the Galactic plane (Turner 1982), but the the narrow line widths (a few $\mathrm{kms}^{-1}$ ) and the similarity in the kinematics and the distribution of extended features and compact maser spots all suggest that the extended $\mathrm{OH}(1720 \mathrm{MHz})$ emission is nonthermal. The significance of extended emission from SN masers is that it is probably tracing the region of shocked molecular gas due to shock chemistry enhancing the abundance of OH molecule (Wardle 1999). Recent work by Wardle et al. (2001) indicates the morphology of extended $\mathrm{OH}(1665$ and $1667 \mathrm{MHz})$ line in absorption is similar to that of extended $\mathrm{OH}(1720 \mathrm{MHz})$ line in emission but with broad linewidths. The distribution of $\mathrm{OH}(1720 \mathrm{MHz})$ line in emission and $\mathrm{OH}(1665$ $\mathrm{MHz}$ ) in absorption associated W28 also is very similar to that of shocked CO cloud identified by Arikawa et al. (1999). 


\section{G359.1-0.5: The Linear Polarization}

G359.1-0.5 is identified as a shell-type SNR with a ring of of CO cloud surrounding it $\mathrm{OH}(1720 \mathrm{MHz})$ observations of this source indicated several compact maser sources at the edge of the remnant having velocities $\sim-5 \mathrm{kms}^{-1}$ (Yusef-Zadeh et al. 1995). We have recently observed this source with the VLA in its A configuration to search for polarized emission from $\mathrm{OH}(1720 \mathrm{MHz})$ masers. Figure 2a shows the spectra of all four Stokes parameters toward the brightest maser (A). Circular polarization due to Zeeman splitting is fitted and the inferred magnetic field along the line of sight is estimated to be $+561 \pm$ $32 \mu \mathrm{G}$. The linearly polarized emission at this wavelength is also detected with a fractional polarization of about $8 \%$. The distribution of the electric field as noted in Fig. $2 \mathrm{~b}$ is is seen predominantly in the direction perpendicular to the edge of the remnant at a position angle of abut $-70^{\circ}$. Theoretical modeling of the magnetic field direction suggests that the B field either aligns with the polarization direction or runs perpendicular to it (Elitzur 1996). It is interesting that recent observations of the $\mathrm{S}(1)$ 1-0 $\mathrm{H}_{2}$ line emission from the edge of the remnant toward the $\mathrm{A}$ maser indicate an elongated $\mathrm{H}_{2}$ feature with the size of $90^{\prime \prime} \times 15^{\prime \prime}$ (length $\times$ width) directed tangential to the shell with a PA $\approx 40^{\circ}$ (Lazendic et al. 2001). The $\mathrm{H}_{2}$ emission is thought to arise from a shock front which is driven by the expansion of the remnant. Assuming that the magnetic field lines are oriented along the shock front and that the $\mathrm{OH}(1720 \mathrm{MHz})$ maser and $\mathrm{H}_{2}$ emission arise from the cooling gas behind a C-type shock, the direction of the magnetic field is then aligned with the long axis of the $\mathrm{H}_{2}$ feature. This implies that the B field ambiguity is resolved and and that it runs perpendicular to the polarization vectors of linearly polarized emission, as noted in Fig. $2 \mathrm{~b}$.

Acknowledgments: We thank M. Elitzur and W. Watson for useful discussions.

\section{References}

\section{References}

Arikawa et al. 1999, PASJ, 51, L7

Elitzur, M. 1996, ApJ, 457, 415

Frail, D.A. et al. 1994, ApJ, 424, L111

Goss, W.M. 1968, ApJS, 15, 131

Jackson et al. 1993, ApJ, 402, 173

Lazendic, J. et al. 2001, in preparation

Leahy, D.A. 1989, ApJ, 216, 193

Lockett, P. et al. 1998, ApJ, 511, 235

Turner, B. 1982, ApJ, 255, L33

Wardle, M. 1999, ApJ, 525, L101

Wardle, M., Lazendic, J. \& Green, A. 2001, in preparation

Yusef-Zadeh, F. et al. 1999, ApJ, 512, 230

Yusef-Zadeh, F. et al. 1995, Science 270, 1801 

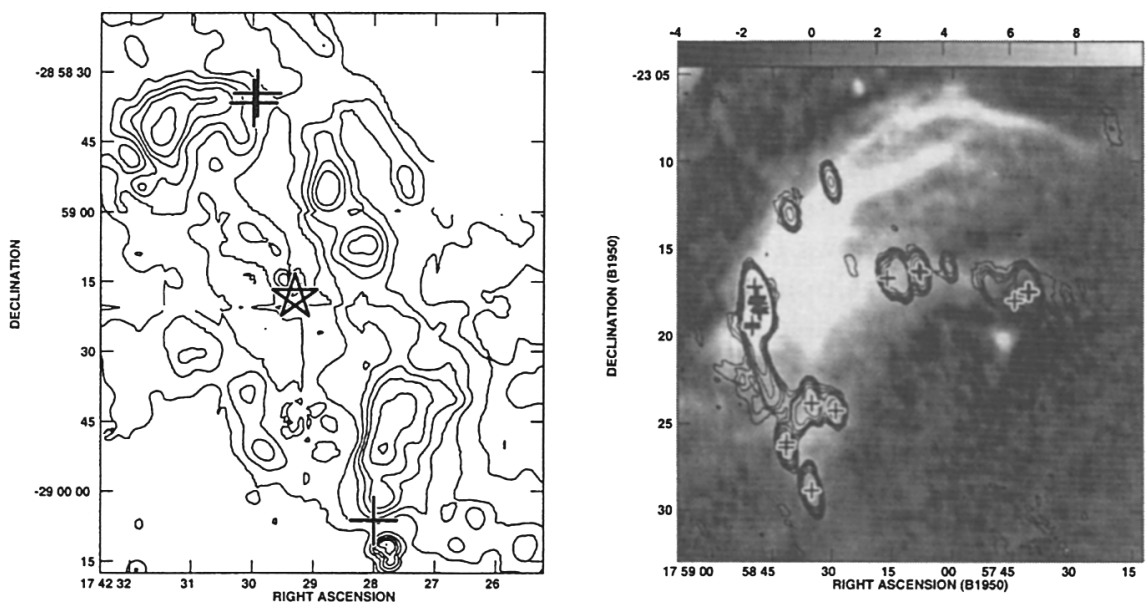

Figure 1. Left panel (Fig. 1a) shows the blue- and red-shifted $\mathrm{OH}$ masers as crosses superposed on the Galactic center molecular ring traced by $\mathrm{H}_{2}$ emission (Yusef-Zadeh et al. 2001, in press). Right panel (Fig. 1b) shows contours of extended emission superposed on the $\lambda 20 \mathrm{~cm}$ continuum image of W28 with a resolution of $28^{\prime \prime} \times 16^{\prime \prime}$.
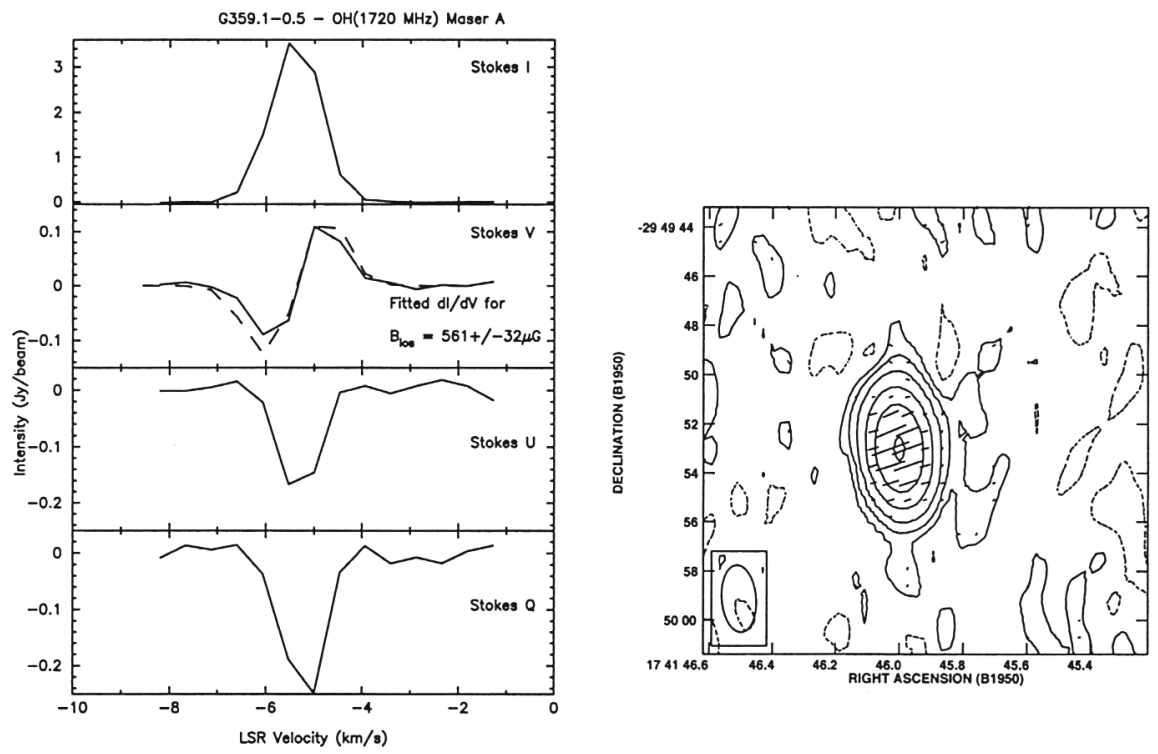

Figure 2. Left panel (Fig. 2a) shows the spectra of all four Stokes parameters toward the brightest maser (A). The distribution of the electric field is shown in the right panel (Fig. 2b) and is seen predominantly in the direction perpendicular to the edge of the remnant. 